\title{
Study on the Development of Shopping Software in the Application of Computer Technology
}

\author{
Yanzhen $\mathrm{Cao}^{1, \text { a }}$ \\ ${ }^{1}$ Zhengzhou University of Industrial Technology, Zhengzhou, Henan, 451100 \\ ${ }^{\mathrm{a}}$ email
}

Keywords: Computer Technology, Application, Shopping Software, Development

\begin{abstract}
Information network is the trend of development of modern society, computer technology is relatively mature led to the development of corresponding industries, shopping software development process fit people's mode of life. Beginning in 2010, shopping cart software has now become a theme software to build shopping site is now the most basic manifestation of e-commerce, to some extent, in terms of software to build a shop for everyone open shopping platform, reduce the face of consumer products sectors, cost savings.
\end{abstract}

\section{Introduction}

Shopping Software development is an important aspect of the application of computer technology, computer technology, the most direct expression of public service. Since 2010, Taobao, Lynx, only products will, Tutu Street shopping software structures, realized directly from the manufacturers and consumers face to achieve a reduced cost shopping, to achieve a quick shopping of.

Computer technology in the process of building the shopping software, tend to have a lot of factors involved, that we need computer technicians to work hard to overcome the existing problems, improve the shopping environment, increase consumer confidence in shopping software.

\section{Overview of Internet Shopping Software}

Online shopping software is now the most close to people's lives of computer technology, software development shop can facilitate people's life style to a certain extent.

The development of online shopping software. Development of science and technology level is the key constraint shopping software development, along with the maturity of network technology, software development shop is now a trend of network technology development. Use of the Internet software development is one of the key factors in the use of network technology is now capable of converting the above cultures use technology to change the constitution change the whole system of factors that will change the culture of the above conversion factors important factor. Shopping software development to facilitate people's lives, with good prospects for development, we can say the future pattern of people's lives depend on that shopping software.

The status of online shopping. Online shopping is the product of high-paced life mode now, in order to facilitate efficient manner now become the first choice of city dwellers to build online shopping platform is now changing a lot of communication modes, as of January 2004 China has Internet users 7950 million , and there are more than $40.7 \%$ of Internet users in the past year there have been online shopping experience, which indicates that Chinese users have online shopping is 3235.7 million, and this number is growing at a rate of about $17 \%$ growth, to in 2006 China's online shopping users will reach 6962 million by 2011 online shopping user size of 187 million people, as of June 2015, China's online shopping user scale reached 417 million people, indicating that China's development of online shopping has good mass based, online shopping is increasingly accepted by all.

China is an important group to use the shopping software development, the development of computer technology in the shopping software, combined with the status of domestic online shopping now is to transform the new model.

Advantages and prospects of development of online shopping system. Online shopping is 
convenient and efficient mode, it is possible to some extent, saving everyone time to shop. Application of Computer Technology in shopping software development, computer technology can make certain services to the public.

The traditional mode of selling goods, need to go through a lot of processes, increased commodity input costs, had led to a dime stuff finally reach the consumer, when you need a dollar.

Application of Computer Technology in shopping software development, avoiding the transfer process, cost savings, and the remaining amount of goods can be directly linked to the Internet, to avoid excess production. Implements accessibility contacting manufacturers and consumers, the manufacturer can know exactly the needs of consumers.

Accelerate the pace of development of China's economy, shopping, software development, in line with the fast-paced life now urbanization, saving people's shopping time shopping software and powerful, able to show the main aspects of its product sales and its sales the details of the change reflects the culture of the use of models.

The traditional mode of marketing requires a variety of programs, requires a variety of certificates, now shopping software shops opened, just simple steps, the program of Simplification. Many are now comfortable shopping online through its own software for the company's product marketing, in the merchandise sales model, timely adjust their business strategies in order to enhance the market competitiveness of their goods.

\section{Application of Computer Technology in the Shopping Software Development Methodology}

Shopping software in our country in recent years began to pop up, foreign mature software development technology compared to the need of our country's computer technology staff to learn, combined with the existing situation of combined development for our domestic shopping software. United States, Britain, Japan and other developed countries, compared to shopping software development, development of shopping software is still in development stage, the need to constantly improve build shopping platform. Now reach for the actual development of the domestic software shopping patterns, computer technology is one of the important support shopping software development tools.

Improve the construction of shopping software background. Need to change the information store timely updates shopping patterns, background build shopping software, is the application of a complex computer technology development, the need for computer technology is mature, improve the shopping software background, the ability to merchandise display look more direct.

Shopping software development on the network through a virtual machine switched network implementations, shopping software structures rely on computer technology, exchange of communication between the client and the Store and resources and between resources and achieve economic modernization.

Requires the government to play the role of macro-control. Shopping software development relies on computer technology matures, but there are flaws in computer technology, which requires the government introduced the law to safeguard the rights of consumers, increase the confidence of consumer spending. Can to a certain extent, changes in consumption patterns of specific changes, once again promote the development of the consumption patterns of the pattern, the pattern will be an important cultural change for cultural change above. Cultural change will carry out the above pattern, cultural trends presented above cultural changes now, can to a certain extent, promoted the important reasons for specific changes in the culture of the above.

Computer technology is a background shopping software support. Computer technology is relatively mature, and now smartphone launch shopping software installed on intelligent machines, mobile APP developed shopping software, such as Taobao this shopping software into mobile shopping software APP development and computer shopping software development, especially in this proof of application of computer technology is relatively mature.

Create a secure online shopping environment. Now China's computer technology is not fully mature, the need for computer technicians in the development of shopping software, but also pay attention to create a secure shopping environment, on the one hand is to protect the interests of 
consumers; another aspect is to increase consumer shopping software trust.

Computer technology needs in the development of shopping software, but also to ensure the development of environmentally safe shopping software, on the other hand the expansion of shopping software.

Expand the promotion of the shopping software. Shopping software that can be known is inseparable from all outstanding marketing programs, shopping software should be combined with the promotion of the corresponding preferential policies to attract all eyes, features such as CD products will highlight the product, using the brand sale. To attract everyone's eye.

Shopping cart software, processes and systematic. Shopping cart software process should be systematic, setting the entire shopping page should follow the concept of humanity, caring for the service hit. Shopping software modules defining, close to the physiological characteristics of the human; to some extent be able to get all the recognition, beautiful software interface settings, appropriate incentives to retain customers.

Diversification shopping software payment. Shopping software payment methods should be combined with the actual situation now, diversified design, barrier-free payment. Now popular alipay balances treasure, flower chanting and other payment platforms, so shopping cart software should these platforms combine to avoid payment difficult problem to some extent.

Application of computer technology in the shopping software, the most notable feature is the ability to understand the situation of the consumers in a timely manner to understand customer demand conditions for commodities to meet the demands of consumers of technology to improve the quality of their products. Shopping software product news show in front of consumers, compared to traditional shopping, shopping, software development, is product specific information, showing out, and even sales, consumers can buy according to their needs and can be ordered at any time, without time and space constraints, from ordering, payment, and then arrived, just by shopping software operation, avoid a lot of procedures, saving time. At the same time, computer technology to promote the diversification of shopping software means of payment, shopping software is now the most efficient way to shop, to some extent, reduce the flow of money through Paypal, money paid through online banking application mode, the original some consumption patterns into the most direct consumption.

\section{Problems of the Computer Technology in the Shopping Software Development}

Shopping cart software in China's model is relatively mature, but there are technical limitations therein. We need more software developers to be able to weigh. Our shopping software platform relatively broad, the one of many, China has a population of a widespread increase in network data resources, and make purchases via shopping software According to $63.7 \%$ of people said that by 2014, China's shopping software deal amount accounted for 2.7889 trillion, shopping software development is a measure of convenience. With the application of computer technology in shopping mode software, is used in a relatively mature, capable of showing a key factor in the transformation of culture.

Computer technology gaps, pages arranged imperfect. Shopping software development relies on computer technology matures, computer calculation tools from the very beginning, and now people can fit a variety of software development community, to a certain extent, proved the maturity of the computer technology. But in the background set some shopping software, there are computer programs set unreasonable, the customer touches added cart menu when the failure to set resource information website above items can not be updated in time, resulting in a customer's bill delay.

The credibility of the shopping software commodity can not be guaranteed. Compared to the traditional mode of shopping is people, people and goods, commodities and commodity exchange model, and now online shopping software is the human face of the commodity, the most direct mode of communication. Shopping software developers in setting commodity information when false situation exists, the information is not updated even shops. Consumers can only understand cart software shops displayed product information, but can not understand the specific credit shops and shopping software disputes in the country's existing laws, there is no clear division of the 
protective measures. Once the process of consumption is a problem, the consumer rights protection is an important issue.

Shopping software just to show product information, the lack of realism. Shopping software show information specific commodities, consumer goods in the selection of hands, to see is to evaluate the product, but it is a lot of black heart of the seller, the use of tamper background sales data, a manufacturing false information to deceive consumers phenomenon. And is now shopping software technology is not fully mature, it is because of computer technology in the development of shopping software, legacy technology gaps.

There is a problem in the development of computer technology background shopping software. Computer technology in the shopping software development, there are significant technical problems, the computer displayed the background data appeared lag data easy for misleading consumers.

The software shopping complex network environment. Shopping software through a network of virtual machines converted from, is now a trend for shopping. Shopping is the public face of software development, to fit the concept of public consumption, in order to highlight the advantages of shopping software.

Computer technology is a relatively broad range of areas, the program of public resources into becoming the most receptive shopping software development model. Shopping software is the use of computer technology, network resources, virtual space, the objective of the show in front of consumers, but too much of the conversion process occurs, which leads to complex network environments.

\section{References}

[1] Zha Jinxiang, Wang Lisheng. empirical study of online shopping satisfaction factors [J]. Management Science. 2006 (01).

[2] Qian Bi. On the computer network marketing model and its characteristics [J]. Chinese commerce. 2011 (25)

[3] Jin Jungang, Huang Yu. Online shopping [J]. Market Modernization. 2008 (28).

[4] Xiao Jie. Character and quality coexistence win hearts and profit both-Scitech actively safeguard the legitimate rights and interests of consumers [J]. Beijing Business Administration, 1997 (03).

[5] online shopping can become the future mainstream way to shop [J]. China Information Review]. 2000 (08).

[6] Wu Yingge. The Problems Existing online shopping [J]. Northern Economy. 2006 (04).

[7] In 2011 online shopping product quality management and risk control forum held in Beijing [J]. Chinese commerce. 2011 (27).

[8] Xu heqing, Jie Xinhua. study the main factors of consumers' online shopping [J]. Consumer Economics. 2009 (02).

[9] Cai Yi. WebGIS-based network traffic information system key technology research and system design [D]. South China University of Technology. 2012.

[10] Huang Kewei. computer network technology in the financial area of Application Status and Problems [J]. Heilongjiang Meteorology. 2012, (03). 